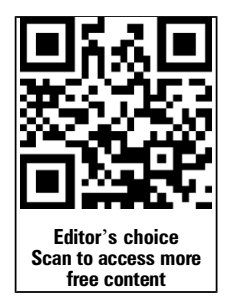

${ }^{1}$ Department of Cell and Molecular Biology, Tulane University, New Orleans, Louisiana, USA

${ }^{2}$ Fourth Affiliated Hospital of China Medical University, Shenyang, Liaoning,

P. R. China

${ }^{3}$ Department of

Ophthalmology, Tulane University, New Orleans, Louisiana, USA

\section{Correspondence to} Dr Shusheng Wang, Department of Cell and Molecular Biology, Department of Ophthalmology, Tulane University, 2000 Percival Stern Hall, 6400 Freret Street, New Orleans, LA 70118, USA; swang1@tulane.edu

Received 6 April 2015 Revised 6 October 2015 Accepted 16 October 2015 Published Online First 9 November 2015

\title{
Current therapeutic developments in atrophic age-related macular degeneration
}

\author{
Jakub Hanus, ${ }^{1}$ Fangkun Zhao, ${ }^{1,2}$ Shusheng Wang ${ }^{1,3}$
}

\begin{abstract}
Age-related macular degeneration (AMD), a degenerative disorder of the central retina, is the leading cause of irreversible blindness in the elderly. The underlying mechanism of the advanced form of dry AMD, also named geographic atrophy (GA) or atrophic AMD, remains unclear. Consequently, no cure is available for dry AMD or GA. The only prevention option currently available is the Age-Related Eye Disease Study (AREDS) formulation, which has been demonstrated to slow down the progression of dry AMD. This review summarises recent advances in therapy for dry AMD and GA. Building on the new understanding of the disease and recent technological breakthroughs, numerous ongoing clinical trials have the goal of meeting the need to cure AMD. Therapeutic agents are being developed to target the key features of the disease, including inhibiting the complement pathway and other inflammatory pathways, reducing oxidative stress and protecting retinal pigment epithelial (RPE) cells, inhibiting lipofuscin and visual cycle, regenerating RPE cells from stem cells and restoring choroidal blood flow. Some of these therapeutic options, especially the stem cell-based therapy, hold great promise, which brings great hope for this devastating blinding disease.
\end{abstract}

\section{INTRODUCTION}

Age-related macular degeneration (AMD) is the leading cause of irreversible blindness in the elderly in the developed world. About $8.7 \%$ of the world's population has AMD, and the number is projected to increase to approximately 196 million in 2020 and to approximately 288 million in $2040 .{ }^{1}$ The direct cost associated with AMD globally is estimated at $\$ 255$ billion. $^{2}$ AMD is characterised by drusen and pigmentation changes in the choroid/ retinal pigment epithelial (RPE) layers in the macula, the region of central retina responsible for fine visual acuity. ${ }^{3}{ }^{4}$ The advanced stage of AMD has dry and neovascular (wet) forms, with no exclusive dichotomy between them. The advanced form of dry AMD is called geographic atrophy (GA) or atrophic AMD. GA is characterised by well-defined areas of RPE loss followed by the degeneration of the corresponding photoreceptors and thinning of the retina. ${ }^{5}$ Neovascular AMD is characterised by choroidal neovascularisation (CNV). Severe central visual impairment or blindness can develop as a result of neovascular or dry AMD. Dry AMD accounts for 85\%-90\% of cases of AMD, and GA is responsible for approximately $20 \%$ of all cases of legal blindness. ${ }^{6}$ Considerable amelioration of neovascular AMD can be achieved with the use of antiangiogenic agents, photodynamic therapy or thermal laser. ${ }^{7-9}$ However, no cure is currently available for dry AMD, and the only preventive option is the Age-Related Eye Disease Study (AREDS) formulations, which reduce the risk of AMD progression by $25 \%-30 \%$ over a 5 -year period. 1011

The etiology of AMD and GA remains elusive, and is believed to result from a combination of factors, including sustained oxidative stress, chronic inflammation, predisposing genetic and environmental factors. ${ }^{12} 13$ Intensive oxygen metabolism, continual exposure to light, high concentrations of polyunsaturated fatty acids, and the presence of photosensitizers increase the production of reactive oxygen species (ROS) in the retina. ROS-induced oxidative stress can cause the induction of programmed necrosis in RPE cells and chronic inflammation, leading to a pathological immune response in AMD (figure 1). ${ }^{14}$ Consistently, knockout of antioxidative genes in mice $\left(\operatorname{Sod} 1^{-/-}\right.$mice, $\operatorname{Sod} 2$ knockdown mice and $\mathrm{Nrf2^{-/- }}$ mice) results in the development of the typical features of AMD. ${ }^{15} 16$ Genetically, polymorphism in a number of genes, including members of the complement pathway, apolipoprotein E (ApoE), ARMS2, HTRA1, CX3CR1, VEGF-A and ABCA4 have been associated with $\mathrm{AMD}$, indicating the involvement of inflammation, lipid metabolism, RPE dysfunction, and angiogenesis in AMD. ${ }^{17}$

Built on a new understanding of the genetics and pathogenesis of AMD, as well as new breakthroughs in stem cell biology, numerous efforts have been focused on developing novel therapy for dry AMD and GA. This review focuses on recent or ongoing clinical trials (summarised in table 1), dividing therapeutic agents into five categories: anti-inflammatory agents, antioxidation and RPE protection agents, lipofuscin and visual cycle inhibitors, choroidal blood flow restoration agents, and stem cell-based therapy. If you are interested in other potential future therapeutics not yet in clinical trials, refer to the recent review by Cunnusamy et al. ${ }^{18}$

\section{CURRENT THERAPEUTIC DEVELOPMENTS FOR GA \\ Anti-inflammatory agents}

Chronic inflammation is believed to play an important role in AMD pathogenesis. $^{19}$ Extracellular drusen deposits, located between the RPE and Bruch's membrane, contain RPE debris (including apolipoprotein E) and inflammatory proteins, such as complement components and complement activators. This suggests the involvement of inflammation in early pathogenesis of AMD. ${ }^{20}$ Drusen accumulation together with oxidative stress can result in RPE cell damage or death, leading to 


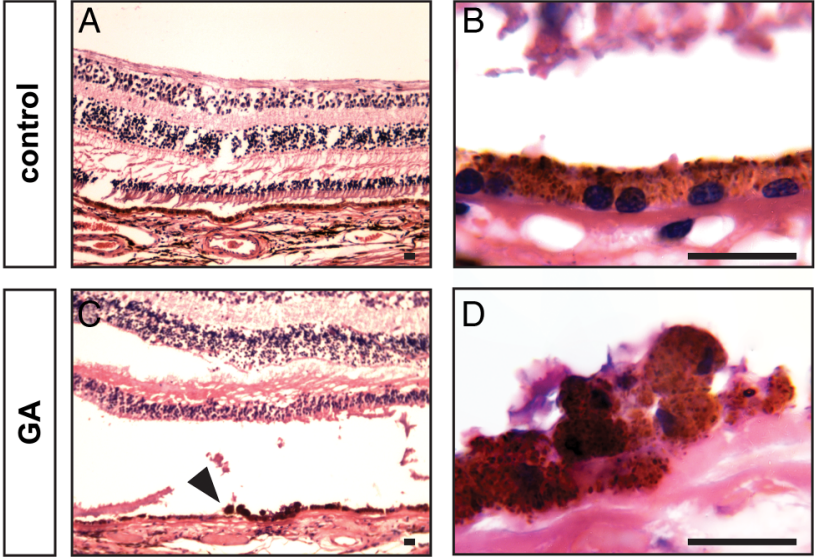

Figure 1 Features of GA in the human samples. Morphologic features of human donor eyes stained with $\mathrm{H} \& \mathrm{E}$. Healthy retina is characterised by the presence of a single layer of pigmented and polarized RPE cells ( $A$ and $B$ ). GA is characterised by RPE cell death and detachment from the basal membrane (C and D). RPE are swollen, characterised by shrunk nuclei and lack of polarisation. Arrowhead indicates transition zone. The visible gap between RPE and photoreceptors in $\mathrm{C}$ is an artefact created during sample preparation. Scale bar: $25 \mu \mathrm{m}$. GA, geographic atrophy; RPE, retinal pigment epithelial.

sustained inflammatory response resulting in the progression of AMD. Polymorphisms in the complement pathway, including complement factors $\mathrm{H}, \mathrm{B}$ and $\mathrm{I}$, and complement components 2, 3, and 7 have been shown to be associated with AMD. ${ }^{21-25}$ Activation of the complement pathway leads to the formation of the membrane attack complex that causes target cell lysis and chemokine release, which in turn can recruit inflammatory cells and increase vascular permeability. ${ }^{26}$ Inhibition of the overactive complement pathway and other inflammatory pathways represents a viable therapeutic approach for halting AMD and GA.

Eculizumab is the first Food and Drug Administration (FDA)-approved complement inhibitor for the treatment of uraemic syndrome. ${ }^{27}$ It is a humanised IgG antibody that inhibits C5 activation. The Complement Inhibition with Eculizumab for the Treatment of Nonexudative Age-Related Macular Degeneration (COMPLETE) phase II trial on eculizumab recruited 60 participants (50 years and older) with dry AMD documented by fundus photography, fundus autofluorescence (FAF) and fluorescein angiography. The first group of patients received a low-dose regimen of $600 \mathrm{mg}$ weekly for 4 weeks followed by $900 \mathrm{mg}$ for every 2 weeks until week 24 . The second group of patients received a high-dose regimen of $900 \mathrm{mg}$ followed by $1200 \mathrm{mg}$ over the same time frame. Primary outcome measured change in drusen volume and GA area at 26 weeks. The study showed that systemic complement inhibition with eculizumab was well tolerated through 6 months, but did not decrease the growth rate of GA or drusen significantly. 2829

Zimura (ARC-1905) is a PEGylated synthesised single-strand nucleic acid aptamer that targets and inhibits complement factor C5 by blocking the cleavage of C5 into C5a and C5b fragments. ${ }^{30}$ Phase I trial for dry AMD evaluated the safety and tolerability of intravitreous Zimura injection in patients with GA secondary to dry AMD. Fifty participants (50 years or older) with dry AMD (drusen and/or GA) in both eyes were recruited. The study was completed with no results being posted, but Ophthotech plans to initiate phase II/III clinical trial investigating Zimura for the treatment of GA.

Lampalizumab is a humanised monoclonal antibody targeting complement factor D in the alternative complement pathway. In a phase II trial, lampalizumab showed a reduction rate of $20.4 \%$ in the GA area at 18 months in patients with advance dry AMD. ${ }^{31}$ Phase III study is currently recruiting participants with estimated enrolment of 936 participants at age 50 years and older. Participants with well-demarcated area or areas of GA secondary to AMD, but no CNV in both eyes will receive a $10 \mathrm{mg}$ dose of lampalizumab administered intravitreally. Primary outcomes will measure change in GA area after 48 weeks, and best corrected visual acuity (BCVA) up to 2 years after the beginning of the study.

Sirolimus (rapamycin), is an immunosuppressive agent that has been approved for preventing organ rejection following

Table 1 Summary of clinical trials targeting geographic atrophy

\begin{tabular}{|c|c|c|c|c|}
\hline Target & Treatment & $\begin{array}{l}\text { Clinical trial } \\
\text { number }\end{array}$ & Company & Status of clinical trials \\
\hline \multirow[t]{5}{*}{ Anti-inflammatory } & Eculizumab & NCT00935883 & Alexion Pharmaceuticals (Cheshire, Connecticut, USA) & Completed phase II \\
\hline & Sirolimus & NCT00766649 & National Eye Institute (Bethesda, Maryland, USA) & Completed phase I/II \\
\hline & Lampalizumab & NCT02247479 & $\begin{array}{l}\text { Hoffmann-LaRoche (Basel, Switzerland) } \\
\text { Roche (Basel, Switzerland) }\end{array}$ & Phase III currently recruiting \\
\hline & $A R C-1905$ & NCT00950638 & Ophthotech (Princeton, New Jersey, USA) & Completed phase I \\
\hline & Glatiramer acetate & NCT00541333 & The New York Eye \& Ear Infirmary (New York, New York, USA) & $\begin{array}{l}\text { Phase I suspended participant } \\
\text { recruitment }\end{array}$ \\
\hline \multirow[t]{2}{*}{ Antioxidants } & AREDS2 & NCT00345176 & National Eye Institute (Bethesda, Maryland, USA) & Phase III completed \\
\hline & OT-551 & NCT00306488 & $\begin{array}{l}\text { National Eye Institute (Bethesda, Maryland, USA) } \\
\text { Other Pharmaceuticals (Exton, Pennsylvania, USA) }\end{array}$ & Phase II completed \\
\hline \multirow{3}{*}{$\begin{array}{l}\text { Visual cycle } \\
\text { inhibitors }\end{array}$} & Fenretinide & NCT00429936 & Sirion Therapeutics, Inc. (Tampa, Florida, USA) & Phase II completed \\
\hline & $\begin{array}{l}\text { Emixustat Hydrochloride } \\
\text { (ACU-4429) }\end{array}$ & NCT01802866 & Acucela Inc. (Seattle, Washington, USA) & Phase II/III ongoing \\
\hline & ALK-001 & NCT02230228 & Alkeus Pharmaceuticals, Inc. (Boston, Massachusetts, USA) & Phase I completed \\
\hline \multirow[t]{3}{*}{ Amyloid beta } & MRZ-99030 & NCT01714960 & Merz Pharmaceuticals GmbH (Dessau-Roßlau, Germany) & Phase I completed \\
\hline & RN6G & NCT01003691 & Pfizer (New York, New York, USA) & Phase I completed \\
\hline & GSK933776 & NCT01342926 & GlaxoSmithKline (Brentford, UK) & Phase II ongoing \\
\hline Choroidal perfusion & MC-1101 & NCT02127463 & MacuCLEAR, Inc. (Plano, Texas, USA) & Phase II/III currently recruiting \\
\hline \multirow[t]{3}{*}{ Stem cell therapy } & MA09-hRPE & NCT01344993 & Ocata Therapeutics (Marlborough, Massachusetts, USA) & Phase I/II currently recruiting \\
\hline & MA09-hRPE & NCT01674829 & CHABiotech Co., Ltd (Seoul, South Korea) & Phase I/lla currently recruiting \\
\hline & HuCNS-SC & NCT01632527 & StemCells, Inc. (Newark, California, USA) & Phase $1 / I I$ ongoing \\
\hline
\end{tabular}

AREDS2, Age Related Eye Disease Study 2; hRPE, retinal pigment epithelial; HuCNS-SC, human central nervous system stem cells. 
renal transplantation and coronary artery restenosis following balloon angioplasty. ${ }^{32}$ It functions by inhibiting the mechanistic target of rapamycin (mTOR) pathway, which can regulate diverse processes, such as cell growth, survival and autophagy. ${ }^{33} 34$ Phase I/II study recruited 11 participants aged 55 years and older to evaluate the safety of sirolimus in patients with GA and if it can help preserve vision in patients. The participants received $440 \mu \mathrm{g}$ subconjunctival injections of sirolimus in the study eye at baseline and every 3 months thereafter. The rate of change in area of GA was evaluated by fundus photography at 24 months and compared with baseline. This clinical study showed that sirolimus injections did not decrease the rate of GA area growth. ${ }^{32} 35$

Copaxone (or glatiramer acetate) functions to induce suppressor $\mathrm{T}$ cells and downregulates inflammatory cytokines. ${ }^{36}$ A phase I trial was designed to test its safety and efficacy in the prevention of GA progression or conversion of dry AMD to neovascular AMD. The recruitment of participants has been suspended with no results posted yet.

\section{Antioxidation and RPE protection agents}

Oxidative stress has been suggested to be a critical component of AMD pathogenesis. ${ }^{37}$ Oxidative stress can lead to the damage and degeneration of the RPE layer, which is crucial for maintaining the homeostasis of the retina. In support of a critical role for oxidative stress in AMD, cigarette smoking, which induces systemic oxidative stress, is a proven risk factor for AMD. ${ }^{37}$ Modified oxidative products, such as carboxyethylpyrrole and malondialdehyde, have been shown to cause inflammatory response and retinal phenotypes in animal models similar to that in AMD. ${ }^{38} 39$ Amyloid- $\beta$, major proinflammatory component of the plaques in Alzheimer's disease, was also observed in drusen and was correlated with the location of degenerating photoreceptors and RPE cells. ${ }^{40}$ On account of these findings, antioxidants and amyloid- $\beta$ inhibitors have been tested in clinical trials for preventing AMD and GA progression.

The previous phase III AREDS trial has shown that nutritional supplements, which contain vitamin $\mathrm{C}$, vitamin $\mathrm{E}, \beta$-carotene, zinc and copper, reduced progression to advanced neovascular AMD by $25 \%$ over the 5 -year study period with no effect on AMD progression to GA. ${ }^{41} 42$ The AREDS2 trial was designed to test whether adding lutein and zeaxanthin (or substituting them for $\beta$-carotene) and/or omega-3 fatty acids could improve the AREDS formulation. This large-scale trial enrolled more than 4000 participants, aged 50-85 years, at risk for advanced AMD. The primary outcome was to determine the development of advanced AMD in people at moderate to high risk of progression. The study found that lutein and zeaxanthin together appeared to be safe and effective alternative to $\beta$-carotene in a median follow-up period of 5 years (IQR, 4.3-5.1 years). ${ }^{43}$ Analyses of the AREDS participants over a 12-year period found that participants with the highest omega-3 fatty acids intake were 30\% less likely than their peers to develop central GA and neovascular AMD. ${ }^{44}$

OT-551 (disubstituted hydroxylamine) is a small molecule with antioxidant and anti-inflammatory properties. It has been shown to protect light-induced RPE degeneration in vivo. ${ }^{45}$ A phase II study has been performed on a small group of 11 participants, aged 60 years and older, with GA present in both eyes. Topical OT-551 (at $0.45 \%$ ) eye drops were given three times a day for up to 3 years. The primary and secondary outcomes included measuring BCVA and fundus autofluorescence (FAF), respectively. The study showed that topical administration of OT-551 was well tolerated but did not exert a significant effect on lesion enlargement, retinal sensitivity or total drusen area. ${ }^{46}$
Currently, there are three clinical trials targeting amyloid- $\beta$ to treat AMD. MRZ-99030, developed as eye drops, is a dipeptide containing d-tryptophan and 2-amino-2-methylpropionic acid designed to modulate aggregation of amyloid- $\beta$. RN6G is a humanised antibody to prevent accumulation of amyloid $\beta-40$ and $\beta-42$ and is delivered by intravenous injections. Both treatments completed phase I studies to assess their safety and tolerability. ${ }^{47}{ }^{48}$ GSK933776 is a humanised monoclonal antibody intended to modulate levels of amyloid- $\beta$. A phase II trial to investigate the safety and efficacy of GSK933776 in patients with GA secondary to AMD is currently ongoing. One hundred and eighty-four patients with GA aged 55 years and older will receive 3,6 or $15 \mathrm{mg} / \mathrm{kg}$ of GSK933776 via intravenous infusion. The primary outcome is to measure the rate of change in GA area from baseline to 12 and 18 months. The secondary outcome is the change in BCVA from baseline to 18 months.

\section{Lipofuscin and visual cycle inhibitors}

Accumulation of lipofuscin and melanolipofuscin granules have been observed at the sites of RPE atrophy in GA eyes and associated with the GA pathogenesis. ${ }^{49} 50$ Lipofuscin consists of products of peroxidation-derived protein modifications and the advanced glycation end products. ${ }^{51}$ Oxygen uptake in lipofuscin leads to formation of singlet oxygen, superoxide anion and hydrogen peroxide. $^{52} \mathrm{~A} 2 \mathrm{E}$, derived from vitamin $\mathrm{E}$, is a byproduct of the visual cycle and a component of lipofuscin. A2E was shown to be phototoxic to RPE cells in vitro. ${ }^{53} 54$ Oxysterols are generated as byproducts of visual cycle by peroxidation of cholesterol, steroid and fatty acids, and metabolised predominantly in RPE cells. Excess accumulation of oxysterols in the lipofuscin pockets or in Bruch's membrane leads to RPE and photoreceptor cell death. It also acts as an attractant for macrophages to induce inflammation. ${ }^{55}$ Because of documented lipofuscin phototoxicity, targeting lipofuscin and the visual cycle has been proposed as an approach for treating AMD.

Fenretinide (RT-101) was shown to effectively block the formation of A2E and other lipofuscin fluorophores in vivo, with no deleterious effects on visual function or retinal morphology. It functions to reduce the circulating levels of retinol and its carrier protein, the retinol binding protein. ${ }^{56} \mathrm{~A}$ phase II study has been performed to determine the efficacy of fenretinide in GA treatment in 245 patients with GA (50-89 years old). The patients received one $100 \mathrm{mg}$ fenretinide softgel capsule per day for 24 months. No significant trend in reducing GA lesion growth rate was observed over the 24 -month period. ${ }^{57}$

Emixustat is a nonretinoid compound that directly modulates the biosynthesis of visual chromophores through inhibition of RPE-specific protein isomerase 65 (RPE65). RPE65 converts all-trans retinal to 11-cis retinal so it can re-enter the visual cycle. ${ }^{58} 59$ Emixustat also binds to RAR/RXR retinoid receptors or antagonise retinoid-binding proteins. Phase II/III clinical trial is ongoing to determine whether orally delivered emixustat hydrochloride (ACU-4429) reduces the rate of GA progression by measuring GA area and BCVA in 480 recruited patients (55 years and older) in a 24-month period.

ALK-001 is a modified form of vitamin A, in which three ordinary hydrogen atoms have been replaced by nonradioactive, heavy hydrogen atoms. The changes embodied in the ALK-001 molecule make it more difficult to form toxic vitamin A dimer (P2E), a byproduct of vitamin A recycling process contributing to lipofuscin formation in RPE cells. ${ }^{60}$ In vitro results showed that ALK-001 caused approximately sevenfold decrease in the formation of toxic vitamin A aggregates. ${ }^{61}$ A phase I study was designed to assess the safety and 
pharmacokinetics of oral ALK-001 capsules in 40 healthy volunteers (21-70 years old) with no results posted yet.

\section{Choroidal blood flow restoration agents}

Choroidal circulation provides the nutrition and removes the wastes from the retina/RPE. ${ }^{62}$ As a consequence of a reduced choroidal blood flow, metabolic wastes accumulate in the photoreceptor cells, Bruch's membrane and RPE cells. Those events can lead to the development of GA. Therefore, improving choroidal blood flow could facilitate the removal of metabolic wastes from RPE, Bruch's membrane and photoreceptor cells to halt AMD disease progression. ${ }^{563}$

MC-1101 is an FDA-approved oral antihypertensive drug. Its active substance, hydralazine, has been shown to increase choroidal blood flow in ocular hypertensive rabbit models and facilitate retinal function recovery following ischaemic insult in rat eyes. ${ }^{64}{ }^{65}$ It also prevents the rupture of the Bruch's membrane and has anti-inflammatory and antioxidative properties. ${ }^{66}$ Phase Ib clinical trial showed that topical instillation of 1\% MC-1101 produced no significant cardiovascular effects or ocular toxicity; no effect on the blood-eye barrier was noted. Phase II/III is ongoing, and 60 patients (50-85 years old, with early to intermediate dry AMD) will receive topical $1 \%$ ophthalmic solution and be assessed for visual function over 24 months.

\section{Stem cell therapy}

Stem cell-based therapy provides new hope for people at risk or suffering from blindness due to degenerative retinal diseases. Replenishing the lost or degenerating RPE cells in GA before the photoreceptors are irrevocably damaged with stem cellderived RPE cells represents the forefront in the practice of regenerative medicine. RPE can be differentiated from human embryonic stem cells (hESCs) or human induced pluripotent stem cells (iPSCs). ${ }^{67-69}$ hESC or human iPSC-derived RPE cells display RPE-like morphology and express typical RPE markers and have the ability to phagocytose photoreceptor segments. When they were transplanted subretinally into rat or mouse model of RPE dysfunction, the grafted cells were retained and retinal function improved. ${ }^{70-73}$

Based on the preclinical data, the first clinical trials investigating the subretinal transplantation of hESC-derived RPE cells have been approved by European and American regulatory authorities. Phase I trials were designed to test the safety and tolerability of grafted hESC-derived RPE cells in patients with either dry AMD or Stargardt's macular dystrophy. ${ }^{74}$ The data showed that transplanted cells persisted at 4 months after transplantation with no signs of rejection or evidence of hyperproliferation or tumourigenesis. In addition, functional recovery was observed in patients receiving hESC-derived RPE. Based on these, two phase I/II trials were performed with 18 patients enrolled. The patients received subretinal injections of 50000 $150000 \mathrm{RPE}$ cells $^{75}$ and followed up for a median of 22 months (12-36 months). Results showed BCVA improvement compared with non-injected fellow eyes, also vision-related quality-of-life had improved in patients with atrophic AMD and Stargardt's disease. This study provided the first evidence of the medium- to long-term safety, survival and biological activity of the stem cell-derived cells in human disease.

There are currently two other clinical trials using hESC-RPE cells (MA09-hRPE). Both are designed to evaluate safety and tolerability of subretinal injection or transplantation of MA09-RPE cells in patients with dry AMD. Both studies are recruiting patients aged 55 years and older, who will receive 50 000-200 000 MA09-hRPE cells. The primary outcome of both studies is to evaluate the safety of hESC-derived RPE cells. Secondary outcomes will measure the mean change of BCVA, autofluorescence photography and reading speed.

In a separate study, clonogenic human central nervous system stem cells (HuCNS-SC) will be evaluated for treatment of dry AMD. ${ }^{76}$ In vivo studies in rats showed that transplantation of the HuCNS-SC cells preserve the number of photoreceptors and their visual function in rodent model of retina degeneration. ${ }^{77} 78$ Phase I/II study will investigate the safety and preliminary efficacy of unilateral subretinal transplantation of HuCNS-SC cells in subjects with GA secondary to AMD.

\section{CONCLUSIONS AND FUTURE DIRECTIONS}

GA is a devastating blinding disease without any cure currently available. However, numerous clinical trials are ongoing with the goal of finding a viable solution to prevent or treat the disease. These therapies target different aspects of GA, including inflammatory pathways, oxidative stress and RPE degeneration, byproducts of the visual cycle, restoration of choroidal perfusion, and replenishing RPE cells with stem cell-derived RPE cells. Some of the trials hold great promise. The AREDS trials show that AREDS formulation reduces the risk of AMD progression by $25 \%-30 \%$, whereas transplanted hESC-derived RPE cells show medium-term and long-term safety, graft survival and possible biological activity shown by improved vision acuity in patients with GA.

Future studies should focus on understanding the pathogenesis of the disease, which remains unclear. The precise delineation of pathological processes within the retina is especially valuable to gain insight of AMD, thus, the correlation between function and morphology play a pivotal role in diagnosing the disease. Clinical studies use FAF imaging to evaluate progressive enlargement of GA. FAF is based on the optical properties of lipofuscin and photoreceptor degeneration products and allows observing lesion areas. The development of advanced imaging system provides state-of-art tools for analysing GA pathophysiology and testing new therapeutics. In this regard, the recent development of spectral domain optical coherence tomography (SD-OCT) and polarisation-sensitive OCT (PS-OCT) technologies allows high-resolution structural imaging of the retinal and subretinal layers, providing powerful methods to quantify GA lesion size and grade GA progression. ${ }^{79-81}$ These new insights in GA morphology using novel retinal imaging strategies are important to design new studies appropriately.

For therapeutic research, it would be critical to test the longterm safety and efficacy of the current hESC- or iPSC-based RPE transplantation approach for GA. Other approaches, including innovative approaches to prevent RPE degeneration, should also be explored. Learning from the clinical trials, going from bedside to the benchside, then back to the bedside could lead to better understanding of the AMD mechanism and better therapy for the disease.

\section{Acknowledgements The authors thank Chastain Anderson for proofreading the manuscript.}

Contributors Jakub Hanus wrote and edited the manuscript. Fangkun Zhao wrote the manuscript. Shusheng Wang wrote and edited the manuscript.

Funding US Department of Health and Human Services > National Institutes of Health > National Eye Institute EY021862. Startup fund from Tulane University. Career development award from the Research to Prevent Blindness foundation and Bright Focus Foundation Award in Age-related Macular Degeneration.

Competing interests None declared.

Provenance and peer review Not commissioned; externally peer reviewed. 


\section{REFERENCES}

1 Wong WL, Su X, Li X, et al. Global prevalence of age-related macular degeneration and disease burden projection for 2020 and 2040: a systematic review and meta-analysis. Lancet Glob Health 2014;2:e106-16.

2 Gordois A, Pezzullo L, Cutler H. The Global economic cost of visual impairment. AMD Alliance International, 2010.

3 Jager RD, Mieler WF, Miller JW. Age-related macular degeneration. N Engl J Med 2008;358:2606-17

4 de Jong PT. Age-related macular degeneration. N Engl J Med 2006;355:1474-85.

5 Holz FG, Strauss EC, Schmitz-Valckenberg S, et al. Geographic atrophy: clinical features and potential therapeutic approaches. Ophthalmology 2014;121:1079-91.

6 Patel HR, Eichenbaum D. Geographic atrophy: clinical impact and emerging treatments. Ophthalmic Surg Lasers Imaging Retina 2015;46:8-13.

7 Brown DM, Kaiser PK, Michels $M$, et al. Ranibizumab versus verteporfin for neovascular age-related macular degeneration. N Eng/ J Med 2006;355:1432-44.

8 Rosenfeld PJ, Brown DM, Heier JS, et al. Ranibizumab for neovascular age-related macular degeneration. N Engl J Med 2006;355:1419-31.

9 Zampros I, Praidou A, Brazitikos P, et al. Antivascular endothelial growth factor agents for neovascular age-related macular degeneration. J Ophthalmol 2012;2012:319728.

10 Age-Related Eye Disease Study 2 Research Group. Lutein + zeaxanthin and omega-3 fatty acids for age-related macular degeneration: the Age-Related Eye Disease Study 2 (AREDS2) randomized clinical trial. JAMA 2013;309:2005-15.

11 Age-Related Eye Disease Study Research Group. A randomized, placebo-controlled, clinical trial of high-dose supplementation with vitamins $C$ and $E$, beta carotene, and zinc for age-related macular degeneration and vision loss: AREDS report no. 8. Arch Ophthalmol 2001;119:1417-36.

12 Beatty S, Koh H-H, Phil M, et al. The role of oxidative stress in the pathogenesis of age-related macular degeneration. Surv Ophthalmol 2000;45:115-34.

13 Khandhadia S, Lotery A. Oxidation and age-related macular degeneration: insights from molecular biology. Expert Rev Mol Med 2010;12:e34.

14 Hanus J, Zhang $H$, Wang $Z$, et al. Induction of necrotic cell death by oxidative stress in retinal pigment epithelial cells. Cell Death Dis 2013:4:e965.

15 Hashizume K, Hirasawa M, Imamura Y, et al. Retinal dysfunction and progressive retinal cell death in SOD1-deficient mice. Am J Pathol 2008;172:1325-31.

16 Zhao Z, Chen Y, Wang J, et al. Age-related retinopathy in NRF2-deficient mice. PLOS ONE 2011:6:e19456.

17 Ding $X$, Patel M, Chan CC. Molecular pathology of age-related macular degeneration. Prog Retin Eye Res 2009;28:1-18.

18 Cunnusamy K, Ufret-Vincenty R, Wang S. Next-generation therapeutic solutions for age-related macular degeneration. Pharm Pat Anal 2012;1:193-206.

19 Telander DG. Inflammation and age-related macular degeneration (AMD). Semin Ophthalmol 2011;26:192-7.

20 Ebrahimi KB, Handa JT. Lipids, lipoproteins, and age-related macular degeneration. J Lipids 2011;2011:802059.

21 Klein RJ, Zeiss C, Chew EY, et al. Complement factor H polymorphism in age-related macular degeneration. Science 2005;308:385-9.

22 Edwards AO, Ritter R III, Abel KJ, et al. Complement factor $\mathrm{H}$ polymorphism and age-related macular degeneration. Science 2005;308:421-4.

23 Haines JL, Hauser MA, Schmidt $\mathrm{S}$, et al. Complement factor $\mathrm{H}$ variant increases the risk of age-related macular degeneration. Science 2005;308:419-21.

24 Gold B, Merriam JE, Zernant J, et al. Variation in factor B (BF) and complement component 2 (C2) genes is associated with age-related macular degeneration. Nat Genet 2006;38:458-62.

25 Yates JR, Sepp T, Matharu BK, et al. Complement C3 variant and the risk of age-related macular degeneration. N Engl J Med 2007;357:553-61.

26 Zipfel PF, Lauer N, Skerka C. The role of complement in AMD. Adv Exp Med Biol 2010;703:9-24

27 Legendre CM, Licht $C$, Muus $P$, et al. Terminal complement inhibitor eculizumab in atypical hemolytic-uremic syndrome. N Engl J Med 2013;368:2169-81.

28 Yehoshua Z, de Amorim Garcia Filho CA, Nunes RP, et al. Systemic complement inhibition with eculizumab for geographic atrophy in age-related macular degeneration: the COMPLETE study. Ophthalmology 2014;121:693-701.

29 Garcia Filho CA, Yehoshua Z, Gregori G, et al. Change in drusen volume as a nove clinical trial endpoint for the study of complement inhibition in age-related macular degeneration. Ophthalmic Surg Lasers Imaging Retina 2014;45:18-31.

30 http://www.ophthotech.com/product-candidates/

31 http://www.roche.com/investors/updates/inv-update-2013-08-27.htm

32 Camardo J. The Rapamune era of immunosuppression 2003: the journey from the laboratory to clinical transplantation. Transplantation proceedings 2003;35(3 Suppl):18s-24s.

33 Laplante M, Sabatini DM. mTOR signaling in growth control and disease. Cell 2012:149:274-93.

34 Hands SL, Proud CG, Wyttenbach A. mTOR's role in ageing: protein synthesis or autophagy? Aging (Albany NY) 2009;1:586-97.

35 Wong WT, Dresner S, Forooghian F, et al. Treatment of geographic atrophy with subconjunctival sirolimus: results of a phase I/II clinical trial. Invest Ophthalmol Vis Sci 2013; $54: 2941-50$
36 Aharoni R. Immunomodulation neuroprotection and remyelination-the fundamental therapeutic effects of glatiramer acetate: a critical review. J Autoimmun 2014;54:81-92

37 Cai $X$, McGinnis JF. Oxidative stress: the Achilles' heel of neurodegenerative diseases of the retina. Front Biosci (Landmark Ed) 2012;17:1976-95.

38 Hollyfield JG, Bonilha VL, Rayborn ME, et al. Oxidative damage-induced inflammation initiates age-related macular degeneration. Nat Med 2008;14:194-8.

39 Suzuki M, Kamei $\mathrm{M}$, Itabe $\mathrm{H}$, et al. Oxidized phospholipids in the macula increase with age and in eyes with age-related macular degeneration. Mol Vis 2007:13:772-8

40 Dentchev T, Milam AH, Lee VM, et al. Amyloid-beta is found in drusen from some age-related macular degeneration retinas, but not in drusen from normal retinas. Mol Vis 2003:9:184-90.

41 Lindblad AS, Lloyd PC, Clemons TE, et al. Change in area of geographic atrophy in the Age-Related Eye Disease Study: AREDS report number 26. Arch Ophthalmol 2009;127:1168-74

42 Chew $E Y$, Clemons TE, Agrón $E$, et al. Long-term effects of vitamins $C$ and $E$, beta-carotene, and zinc on age-related macular degeneration: AREDS report no. 35. Ophthalmology 2013;120:1604-11.e4.

43 Chew EY, Clemons TE, Sangiovanni JP, et al. Secondary analyses of the effects of lutein/zeaxanthin on age-related macular degeneration progression: AREDS2 report No. 3. JAMA Ophthalmol 2014;132:142-9.

44 Sangiovanni JP, Agron E, Meleth AD, et al. \{omega\}-3 Long-chain polyunsaturated fatty acid intake and 12-y incidence of neovascular age-related macular degeneration and central geographic atrophy: AREDS report 30, a prospective cohort study from the Age-Related Eye Disease Study. Am J Clin Nutr 2009;90:1601-7

45 Tanito M, Li F, Anderson RE. Protection of retinal pigment epithelium by OT-551 and its metabolite TEMPOL-H against light-induced damage in rats. Exp Eye Res 2010;91:111-14

46 Wong WT, Kam W, Cunningham D, et al. Treatment of geographic atrophy by the topical administration of OT-551: results of a phase II clinical trial. Ophthalmol Vis Sci 2010:51:6131-9.

47 Parsons CG, Ruitenberg M, Freitag CE, et al. MRZ-99030-A novel modulator of Abeta aggregation: I-Mechanism of action (MoA) underlying the potential neuroprotective treatment of Alzheimer's disease, glaucoma and age-related macular degeneration (AMD). Neuropharmacology 2015;92:158-69.

48 http://www.reviewofophthalmology.com/content/d/retinal_insider/c/44883/

49 Holz FG, Bellman C, Staudt S, et al. Fundus autofluorescence and development of geographic atrophy in age-related macular degeneration. Invest Ophthalmol Vis Sci 2001:42:1051-6.

50 von Ruckmann A, Fitzke FW, Bird AC. Fundus autofluorescence in age-related macular disease imaged with a laser scanning ophthalmoscope. Invest Ophthalmol Vis Sci 1997:38:478-86.

51 Schutt F, Bergmann M, Holz FG, et al. Proteins modified by malondialdehyde, 4-hydroxynonenal, or advanced glycation end products in lipofuscin of human retinal pigment epithelium. Invest Ophthalmol Vis Sci 2003;44:3663-8.

52 Rozanowska M, Jarvis-Evans J, Korytowski W, et al. Blue light-induced reactivity of retinal age pigment. In vitro generation of oxygen-reactive species. J Biol Chem 1995;270:18825-30.

53 Rozanowska M, Pawlak A, Rozanowski B, et al. Age-related changes in the photoreactivity of retinal lipofuscin granules: role of chloroform-insoluble components. Invest Ophthalmol Vis Sci 2004;45:1052-60.

54 Mathieu JM, Schloendorn J, Rittmann BE, et al. Medical bioremediation of age-related diseases. Microb Cell Fact 2009:8:21.

55 Javitt NB, Javitt JC. The retinal oxysterol pathway: a unifying hypothesis for the cause of age-related macular degeneration. Curr Opin Ophthalmol 2009;20:151-7.

56 Radu RA, Han Y, Bui TV, et al. Reductions in serum vitamin A arrest accumulation of toxic retinal fluorophores: a potential therapy for treatment of lipofuscin-based retinal diseases. Invest Ophthalmol Vis Sci 2005;46:4393-401.

57 Mata NL, Lichter JB, Vogel R, et al. Investigation of oral fenretinide for treatment of geographic atrophy in age-related macular degeneration. Retina (Philadelphia, Pa) 2013:33:498-507.

58 Kubota R, Boman NL, David R, et al. Safety and effect on rod function of ACU-4429, a novel small-molecule visual cycle modulator. Retina (Philadelphia, Pa) 2012;32:183-8

59 Kubota R, Al-Fayoumi S, Mallikaarjun S, et al. Phase 1, dose-ranging study of emixustat hydrochloride (ACU-4429), a novel visual cycle modulator, in healthy volunteers. Retina (Philadelphia, Pa) 2014;34:603-9.

60 http://www.ffb.ca/research/research_news/Alkeus_Stargardt.html

61 http://www.alkeuspharma.com/preclinical.html

62 Booij JC, Baas DC, Beisekeeva J, et al. The dynamic nature of Bruch's membrane. Prog Retin Eye Res 2010;29:1-18.

63 Chiou G. Is dry AMD treatable? A new ophthalmic solution may halt disease progression. Retina Today 2012:69-79.

64 Wei Jiang CG. Effects of hydralazine on ocular blood flow and laser-induced choroidal neovascularization. Int J Ophthalmol 2009:2:324-7. 
65 Jiang W, Chiou GCY. Effects of hydralazine on ocular blood flow laser-induced choroidal neovascularization. Int I Ophthalmol 2009;2:324-7.

66 Holz FG, Pauleikhoff D, Spaide RF, et al. Age-related Macular Degeneration. Springer Science \& Business Media, 2012.

67 Carr AJ, Vugler AA, Hikita ST, et al. Protective effects of human iPS-derived retinal pigment epithelium cell transplantation in the retinal dystrophic rat. PLOS ONE 2009;: $4: 8152$.

68 Cho MS, Kim SJ, Ku SY, et al. Generation of retinal pigment epithelial cells from human embryonic stem cell-derived spherical neural masses. Stem Cell Res 2012:9:101-9.

69 Buchholz DE, Hikita ST, Rowland TJ, et al. Derivation of functional retinal pigmented epithelium from induced pluripotent stem cells. Stem Cells 2009;27:2427-34.

70 Kokkinaki M, Sahibzada N, Golestaneh N. Human induced pluripotent stem-derived retinal pigment epithelium (RPE) cells exhibit ion transport, membrane potential, polarized vascular endothelial growth factor secretion, and gene expression pattern similar to native RPE. Stem Cells 2011;29:825-35.

71 Zhu D, Deng X, Spee C, et al. Polarized secretion of PEDF from human embryonic stem cell-derived RPE promotes retinal progenitor cell survival. Invest Ophthalmol Vis Sci 2011:52:1573-85.

72 Vugler A, Carr AJ, Lawrence J, et al. Elucidating the phenomenon of HESC-derived RPE: anatomy of cell genesis, expansion and retinal transplantation. Exp Neurol 2008;214:347-61.

73 Carr AJ, Vugler A, Lawrence J, et al. Molecular characterization and functional analysis of phagocytosis by human embryonic stem cell-derived RPE cells using a novel human retinal assay. Mol Vis 2009;15:283-95.
74 Becker S, Jayaram H, Limb GA. Recent advances towards the clinical application of stem cells for retinal regeneration. Cells 2012;1:851-73.

75 Schwartz SD, Regillo CD, Lam BL, et al. Human embryonic stem cell-derived retinal pigment epithelium in patients with age-related macular degeneration and Stargardt's macular dystrophy: follow-up of two open-label phase 1/2 studies. Lancet 2015;385:509-16.

76 Uchida N, Buck DW, He D, et al. Direct isolation of human central nervous system stem cells. Proc Natl Acad Sci USA 2000;97:14720-5.

77 Cuenca N, Fernandez-Sanchez L, McGill TJ, et al. Phagocytosis of photoreceptor outer segments by transplanted human neural stem cells as a neuroprotective mechanism in retinal degeneration. Invest Ophthalmol Vis Sci 2013;54: $6745-56$.

78 McGill TJ, Cottam B, Lu B, et al. Transplantation of human central nervous system stem cells-neuroprotection in retinal degeneration. Eur I Neurosci 2012:35:468-77.

79 Sayegh RG, Zotter S, Roberts PK, et al. Polarization-sensitive optical coherence tomography and conventional retinal imaging strategies in assessing foveal integrity in geographic atrophy. Invest Ophthalmol Vis Sci 2015;56:5246-55.

80 Sayegh RG, Kiss CG, Simader C, et al. A systematic correlation of morphology and function using spectral domain optical coherence tomography and microperimetry in patients with geographic atrophy. Brit I Ophthalmol 2014;98: 1050-5.

81 Simader C, Sayegh RG, Montuoro A, et al. A longitudinal comparison of spectral-domain optical coherence tomography and fundus autofluorescence in geographic atrophy. Am J Ophthalmol 2014;158:557-66. 


\section{Correction: Current therapeutic developments in atrophic age-related macular degeneration}

Hanus J, Zhao F, Wang S. Current therapeutic developments in atrophic age-related macular degeneration. Br J Ophthalmol 2016;100:122-7. In the first paragraph of the "Lipofuscin and visual cycle inhibitors" section of the paper (p. 124), the authors state "A2E, derived from vitamin E, is a byproduct of the visual cycle and a component of lipofuscin". This sentence should read: "A2E, derived from vitamin A, is a byproduct of the visual cycle and a component of lipofuscin”.

Br J Ophthalmol 2016;100:744. doi:10.1136/bjophthalmol-2015-306972corr1

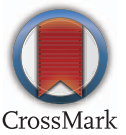

